\title{
Delayed diagnosis of arterial hypertension in young woman with coarctation of the aorta coexisting with arteria lusoria
}

\author{
Ilona Michałowska ${ }^{1}$, Małgorzata Kowalczyk ${ }^{2}$, Marcin Demkow ${ }^{3}$, Piotr Hoffman ${ }^{2}$ \\ ${ }^{1}$ Department of Radiology, Institute of Cardiology, Warsaw, Poland \\ ${ }^{2}$ Department of Congenital Heart Diseases, Institute of Cardiology, Warsaw, Poland \\ ${ }^{3}$ Department of Coronary and Structural Heart Diseases, Institute of Cardiology, Warsaw, Poland
}

\begin{abstract}
Coarctation of the aorta (CoAo) coexisting with an aberrant right subclavian artery (arteria lusoria) is a rare congenital malformation which can be undiagnosed until adulthood. Presented herein, is the case of a 35-year-old woman in her fifth pregnancy with arterial hypertension diagnosed 2 months earlier. The patient had had 4 natural childbirths. She had suffered from palpitations and a transient episode of aphasia. Blood pressure in the right arm was $102 / 71 \mathrm{mmHg}$, in the left arm $166 / 85 \mathrm{mmHg}$, and in the lower extremities $95 / 70 \mathrm{mmHg}$.

The suspicion of CoAo was confirmed by transthoracic echocardiogram. Continuous wave Doppler discovered a substantial pressure gradient across the descending aorta with a characteristic prolonged diastolic phase (Fig. 1A) with concomitant flattened flow encompassing diastolic phase within the abdominal aorta (Fig. 1B).
\end{abstract}

Computed tomography angiography (CTA) of the aorta performed after the last childbirth confirmed the diagnosis of CoAo and revealed arteria lusoria (Fig. 1C, arrow) arising distal to the narrowing of the descending aorta. Extensive asymmetrical collateral circulation on the chest wall and on the right side of the neck (Fig. 1D, E, arrows) was demonstrated. The patient underwent successful treatment by balloon angioplasty with stent placement (Fig. 1F, H). Subsequently, CTA of the carotid arteries showed normal blood circulation in the neck (Fig. 1F). Doppler study showed a restored normal flow pattern within the abdominal aorta (Fig. 1G).

The present case emphasizes a pivotal role of CTA in diagnosing CoAo and associated arterial lesions. CoAo should be considered when diagnosing arterial hypertension in younger patients to bring more attention to this condition.

Conflict of interest: None declared

Address for correspondence: Ilona Michałowska, MD, Department of Radiology, Institute of Cardiology, ul. Alpejska 42, 04-628 Warszawa, Poland, tel: +48 2234341 68, e-mail: imichalowska@ikard.pl 


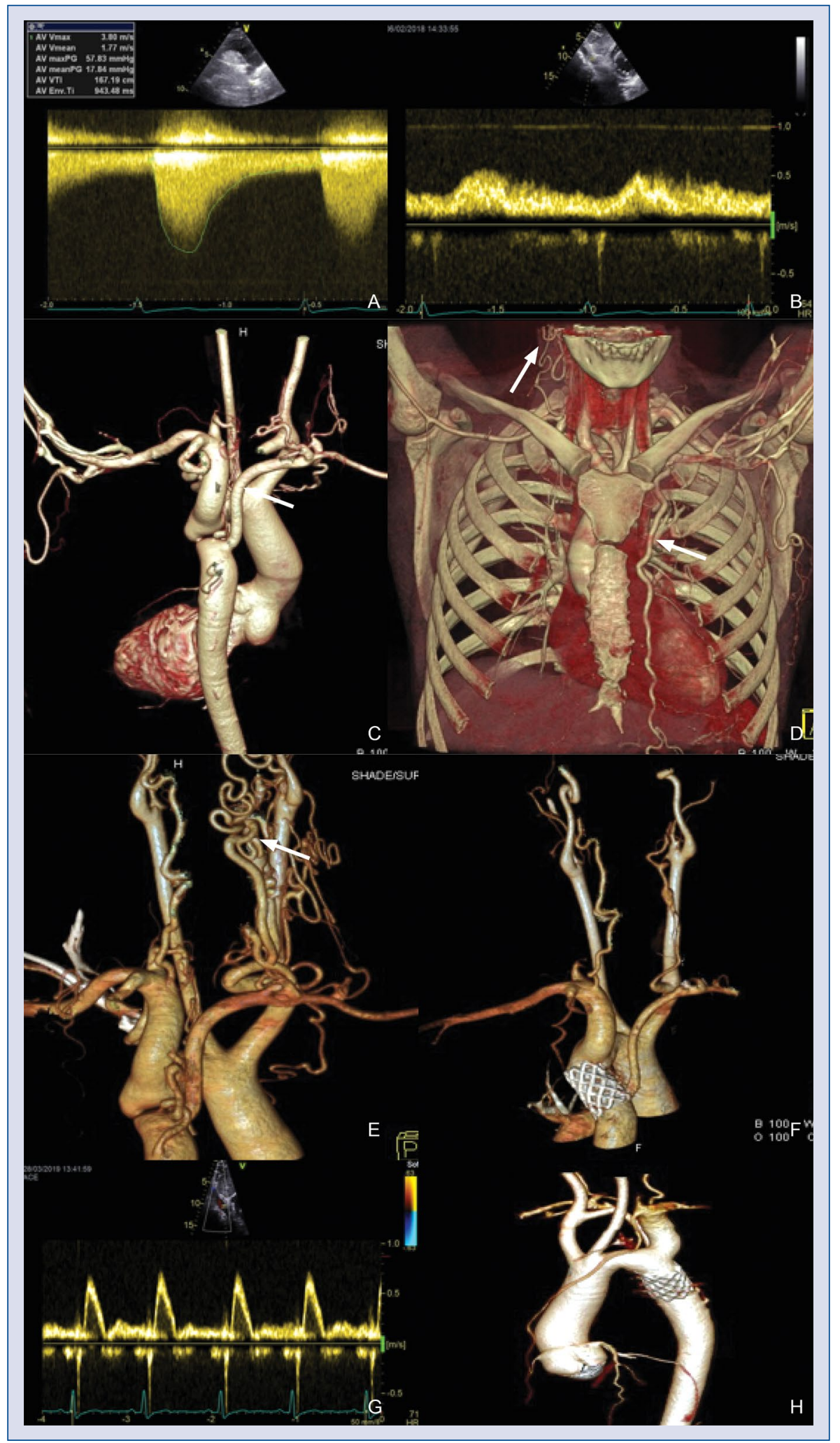

Figure 1. A. Continuous wave (CW) Doppler at coarctation site; B. CW Doppler in the abdominal aorta; C. Computed tomography angiography (CTA), posterior view - coarctation of the aorta, arteria lusoria (arrow); D. CTA - collateral circulation on the chest wall (left side - arrow) and in the neck (right side - arrow); E. CTA, posterior view — extensive asymmetrical collateral circulation on the neck (arrow); F. CTA after stent implantation (posterior view) — normal blood circulation on the neck; G. CW Doppler in abdominal aorta after coarctation treatment; H. CTA of thoracic aorta after stent placement. 